Check for updates

Faculty of Public Health

president@fph.org.uk

Cite this as: BMJ 2020;370:m3553

http://dx.doi.org/10.1136/bmj.m3553

Published: 14 September 2020

\section{Covid-19: An efficient and effective test trace regime is not a numbers game}

\section{We need a targeted testing strategy, not a blunderbuss, say Maggie Rae and Ellis Friedman Maggie Rae, Ellis Friedman}

The government's “moonshot” plan to test millions of people daily for covid-19 risks repeating the mistakes of the early days of test and trace.

The ambition to deliver a further substantial increase in testing is welcome, but as the push for 100000 daily tests exposed, an efficient and effective test and trace regime is not a numbers game. Testing is not a medical intervention and on its own does nothing to control the disease. It only has value if the test is reliable and a positive test triggers a quick and effective response, which means immediately tracing the contacts of the infected person, investigating the source of their infection, and effectively preventing further transmission of the virus.

Identifying large numbers of asymptomatic carriers has the potential to significantly strengthen our ability to manage the disease, but-as the continuing problems with laboratory capacity demonstrate-we are unlikely ever to have the capacity and public compliance to allow us to repeatedly test millions of asymptomatic people and then report the results and trace contacts efficiently. Even in areas where there are major outbreaks, such as Bolton, randomly offering tests to the public will not work effectively and will waste valuable resources. We need a targeted testing strategy, which is part of a well designed control strategy-not a blunderbuss.

An effective test and trace system needs to move fast, be led by local intelligence, and prioritise those groups and settings where the virus can spread quickly-especially to the vulnerable.

The speed of response to local outbreaks is currently being inhibited by excessive central control of the testing resources. Local government public health directors, working with the regional health protection teams of what will be the National Institute for Health Protection, need to be able to call in testing services without the inevitable delays caused by a national system delivered by a contractor answerable to ministers.

The folly of running test and trace primarily as a national system can be seen in the ludicrous situation of people being told to drive many miles to get a test. As well as undermining the effectiveness of the entire covid-19 response, this failure to make testing centres accessible hits the poorest communities hardest, exposing them to even greater risks.

The government needs to prioritise testing. As capacity increases it should expand the scope of testing in a systematic manner. Symptomatic people must be a higher priority than asymptomatic testing. Asymptomatic testing needs to be prioritised to settings which are high risk, like care homes and hospitals, and to settings where compliance with testing is practical and will provide assurance that a setting is safe, such as universities, schools, and perhaps some large workplaces. The risk at universities, when there is evidence that many young people are not following government guidance on social distancing, is clear. The current policy at ports of entry must be reviewed and, when capacity allows, testing should be offered in a system which encourages public compliance.

Beyond this, people in high risk occupations such as bus drivers and minicab drivers should be tested regularly, although this must not be seen as an alternative to ensuring staff are "covid-19 secure" at work.

Big numbers will count for little without the right strategy. We don't need to shoot for the moon, we simply need to follow tried and tested techniques for controlling infectious diseases.

The most hazardous phase of the pandemic is still ahead, with the difficulty of adapting to constantly changing messages, the need to control the virus as we open up the economy, with schools and universities restarting, and as we approach winter flu season. A clear, robust plan is essential if we are to get through the winter without further substantial loss of life.

Competing interests: None declared.

Commissioned, not peer reviewed. 